\title{
Extended Drude model and role of interband transitions in the mid-infrared spectra of pnictides
}

\author{
L. Benfatto, ${ }^{1}$ E. Cappelluti, ${ }^{2,1}$ L. Ortenzi, ${ }^{3}$ and L. Boeri ${ }^{3}$ \\ ${ }^{1}$ Institute for Complex Systems (ISC), CNR, U.O.S. Sapienza and \\ Department of Physics, Sapienza University of Rome, P.le A. Moro 2, 00185 Rome, Italy \\ ${ }^{2}$ Instituto de Ciencia de Materiales de Madrid, ICMM-CSIC, Cantoblanco, E-28049 Madrid, Spain \\ ${ }^{3}$ Max-Planck-Institut für Festkörperforschung, Heisenbergstraße 1, D-70569 Stuttgart, Germany
}

(Dated: May 30, 2018)

\begin{abstract}
We analyze the outcomes of an extended-Drude-model approach to the optical spectra of pnictides, where the multiband nature of the electronic excitations requires a careful analysis of the role of interband processes in the optical conductivity. Through a direct comparison between model calculations of the intraband optical spectra and experimental data, we show that interband transitions, whose relevance is shown by first-principle calculations, give a non negligible contribution already in the infrared region. This leads to a substantial failure of the extended-Drude-model analysis on the measured optical data without subtraction of interband contributions.
\end{abstract}

Optical studies are a useful experimental probe to analyze the interactions at play in different classes of correlated materials,$\underline{1}$ for which it would be desirable to devise a common theoretical scheme. A typical example is the so-called extended-Drude-model (EDM) analysis, where the experimental optical conductivity is analyzed in terms of a Drude-like model with a frequencydependent inverse lifetime and effective mass $\stackrel{\underline{1}}{\underline{t}}$ This approach is well justified for a single-band system interacting with a continuum of bosonic excitations (such for instance phonons in a metal) where it can provide useful information on the relevant collective modes interacting with the electronic particle-hole excitations. 2 The EDM has been widely used also for strongly correlated materials, such as the cuprate superconductors; here, intraband excitations are well separated from the optical interband transitions, which pose a natural cut-off (typically of order of $4000 \mathrm{~cm}^{-1}$ ) to the applicability of the EDM itself in these compounds. .2 .3

After the discovery of superconductivity in iron-based superconductors an intense experimental and theoretical research has been devoted to the EDM analysis in these materials as well $\underline{\underline{4}-8}$ A general outcome of this analysis is a relatively large frequency-dependent in-plane inverse lifetime $\tau^{-1}(\omega) \sim \omega, \frac{4,5}{, 5}$ that, within the EDM, is interpreted in terms of a strong-coupling regime $(\lambda \simeq 3-4)$ for the relevant bosonic excitations,,$\frac{5,6}{6}$ located around 20 $60 \mathrm{meV}$. These are usually identified with the spin fluctuations between the hole and electron pockets of the Fermi surface, that from the very beginning have been suggested as the most promising candidates for the superconducting pairing.$\underline{?}$

There are however two main open issues concerning these results. On one hand, a strong coupling to these low-energy bosonic modes would imply also an effective mass at low energy much larger than that measured by

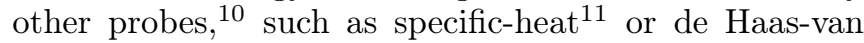
Alphen (dHvA) $\stackrel{12}{=}$ On the other hand, the use of the EDM implicitly assumes that the interband transitions in pnictides become relevant only above a threshold fre- quency of about $\sim 2000 \mathrm{~cm}^{-1}$, even though some authors suggested that they are present at lower energies $\underline{13}-15$ Low-energy interband transitions may invalidate the conclusions of the EDM analysis, introducing spurious effects.

In this paper, we quantify the relative role of interband and intraband transitions in the EDM analysis of the in-plane optical conductivity in pnictides, focusing on $\mathrm{LaFePO}$ where no magnetic transition occurs and where the coupling to spin fluctuations within a multiband Eliashberg approach has been already discussed in the context of different physical properties $\frac{16,17}{17}$ Through a direct comparison with the experimental optical data of Ref. [4] we show that, for the physical range of the coupling of electrons with spin-fluctuations, the intraband contribution alone is not sufficient to account for the large inverse lifetime obtained by the EDM analysis. In this context we also show that low-energy interband transitions, individuated through first-principles calculations, play a dominant role here already at very low energy $\left(\omega \gtrsim 500 \mathrm{~cm}^{-1}\right)$, invalidating the conclusion of a simple EDM analysis of the data. Such a result, along with the similarity of the band structures and optical spectra of Fe pnictides, suggests that a new approach is needed in order to analyze the optical data of pnictides.

The EDM analysis is based on the fact that manybody interactions modify the Drude formula for the optical conductivity by introducing a frequency dependence into the inverse lifetime $\tau^{-1}(\omega)$ and a mass enhancement factor $m^{*}(\omega) / m_{b}$, where $m_{b}$ is the band mass of the carriers. $\frac{1}{2}$ Deconvoluting the real $\sigma_{1}(\omega)$ and imaginary $\sigma_{2}(\omega)$ part of the complex optical conductivity, one can extract:

$$
\begin{aligned}
\tau^{-1}(\omega) & =\frac{\omega_{P}^{2}}{4 \pi} \frac{\sigma_{1}(\omega)}{\sigma_{1}^{2}(\omega)+\sigma_{2}^{2}(\omega)}, \\
\frac{m^{*}(\omega)}{m} & =\frac{\omega_{P}^{2}}{4 \pi \omega} \frac{\sigma_{2}(\omega)}{\sigma_{1}^{2}(\omega)+\sigma_{2}^{2}(\omega)},
\end{aligned}
$$

where $\omega_{P}$ is the plasma frequency, that is usually estimated integrating the real part of the conductivity up to 

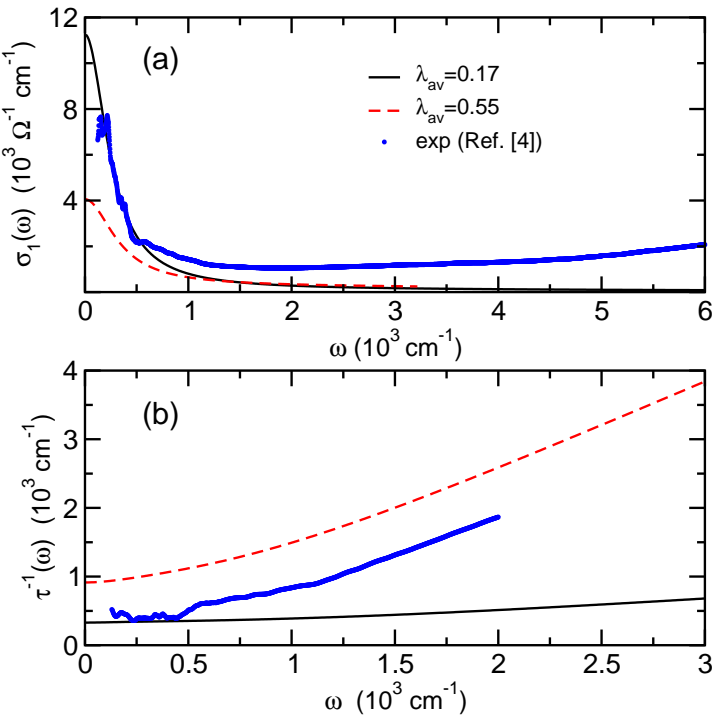

FIG. 1: (color online) Comparison between experimental data (filled circles, after Ref. 4) and theoretical calculations of the intraband contribution (lines) for the optical conductivity (panel a) and the corresponding $\tau^{-1}(\omega)$ as extracted by an EDM analysis (panel b). Different values of the average coupling strength are here considered (see text).

a cut-off $\omega_{c}$. The underlying idea is that the data below $\omega_{c}$ represent intraband optical transitions of interacting charge carriers (with spectral weight proportional to $\omega_{P}^{2}$ ), while the spectrum above $\omega_{c}$ represents interband transitions. In the case of pnictides $\omega_{c}$ is usually assumed around $2000-3000 \mathrm{~cm}^{-1} \underline{\underline{4}} \underline{\underline{\underline{8}}}$

Experimental data for $\sigma_{1}(\omega)$ and for the resulting $\tau^{-1}(\omega)$ in LaFePO, from Ref. 4, are shown in Fig. 1 as blue symbols. In particular, the analysis of $\tau^{-1}(\omega)$ clearly reveals two distinct regimes which are also visible in $\sigma_{1}(\omega)$ : a low-energy one below $\sim 500 \mathrm{~cm}^{-1}$, where $\tau^{-1}(\omega)$ is roughly constant $\left(\tau^{-1}(\omega) \approx 400 \mathrm{~cm}^{-1}\right)$ and which corresponds to a very narrow Drude-like contribution in $\sigma_{1}(\omega)$; and a higher-energy regime $(\omega>500$ $\mathrm{cm}^{-1}$ ) where $\sigma_{1}(\omega)$ is almost constant and $\tau^{-1}(\omega)$ has a steady increase, with $\tau^{-1}(\omega) \sim \omega$. Note that scattering by impurities leads to a constant $\tau^{-1}(\omega)$, while scattering by retarded interactions is usually reflected in an increase of $\tau^{-1}(\omega)$ up a saturation regime at frequencies larger than the typical energy scale of the interaction ${ }^{2}$ Since phonon frequencies in pnictides do not exceed 500 $\mathrm{cm}^{-1}, \frac{19}{19}$ one would be tempted to interpret the lack of saturation in $\tau^{-1}(\omega)$ as due to strong scattering by some other collective modes, $\stackrel{4}{\underline{4}}$ such as spin fluctuations, that are also the best candidates for the superconducting pairing.

To investigate the possible role of spin fluctuations on the optical spectra of LaFePO we calculated the intraband optical conductivity of the fully interacting multiband model for LaFePO already introduced in Refs. 16, 17]. In particular, the charge carriers inter- act with spin fluctuations modeled in terms of a Millis's spectrum,, 20

$$
B_{\mathrm{sf}}(\omega)=\lambda_{\mathrm{av}} \frac{\omega \omega_{0}}{\pi\left(\omega_{0}^{2}+\omega^{2}\right)},
$$

which emphasizes the survival of bosonic excitations up to large energies. Here $\omega_{0}$ is the characteristic energy scale of spin fluctuations and $\lambda_{\mathrm{av}}$ is an average of the dimensionless coupling constant in each of the four bands included in the microscopic model ${ }^{16,17}$ This has been estimated to be $\lambda_{\mathrm{av}} \approx 0.5$ at $T_{c}=7 \mathrm{~K}, 16$ to account for the superconducting, $\stackrel{21}{\Perp}$ magnetic, 22 and specific heat measurements $\stackrel{23,24}{2}$ This coupling is expected to be reduced at $T=300 \mathrm{~K}$ by roughly a factor $3, \lambda_{\mathrm{av}} \approx 0.17$, due to the weakening of the antiferromagnetic correlations, accompanied also by a corresponding shift at higher energies of the characteristic energy scale $\omega_{0}=60 \mathrm{meV} \approx 480$ $\mathrm{cm}^{-1}$ of the spin fluctuations $\stackrel{17,18}{\underline{10}}$ The complex optical conductivity is then computed using a finite quasiparticle scattering rate $\Gamma_{0}=10 \mathrm{meV} \approx 81 \mathrm{~cm}^{-1}$ to account for impurities, and an EDM is applied to these data to extract $\tau^{-1}(\omega)$, using the experimental plasma frequency $\omega_{P}=14900 \mathrm{~cm}^{-1} \underline{\underline{4}}$ The results for $\sigma_{1}(\omega)$ and $\tau^{-1}(\omega)$ obtained in this way are shown in Fig. 11 as solid black lines, in comparison with the experimental data of Ref. 4. As one can see, while the low-energy part of $\sigma_{1}(\omega)$ is well described by the calculated intraband optical conductivity for $\lambda_{\mathrm{av}}=0.17$, at $\omega \gtrsim 500 \mathrm{~cm}^{-1}$ the theoretical $\sigma_{1}(\omega)$ deviates from the experimental data. This behavior is reflected also in the corresponding theoretical $\tau^{-1}(\omega)$, which increases approximately linearly as $2 \lambda_{\mathrm{av}} \omega$, i.e with a slope about four times smaller than the experimental one. Indeed, within this framework, we are able to reproduce the experimental linear slope of $\tau^{-1}(\omega)$ at $\omega \sim 1500 \mathrm{~cm}^{-1}$ only by assuming a total coupling of the order of $\lambda_{\mathrm{av}} \approx 0.55$ (red dashed line in Fig. 1). However such a large value of $\lambda_{\mathrm{av}}$, besides being at odds with the $\mathrm{dHvA}$ and other thermodynamical measurements, $\stackrel{10,16,17}{\Perp}$ would result in a $\tau^{-1}(\omega=0)$, at $T=300 \mathrm{~K}$, much higher than the experimental one. Furthermore, $\sigma_{1}(\omega)$, turns out to be too broad in the low-frequency range with respect to experimental data. The present results show thus that in $\mathrm{LaFePO}$ it is not possible to describe the optical data in the range $\omega \lesssim 2000 \mathrm{~cm}^{-1}$ in terms of pure intraband transitions, even in the presence of scattering mediated by a spin-fluctuation spectrum with a long tail at high energies as in Eq. (3), and regardless the specific value of the coupling.

Since a model with intraband transitions alone cannot reproduce the optical data in pnictides, we investigate the possibility that the EDM analysis of the experimental data in LaFePO is affected by interband transitions having a sizable spectral weight at energies comparable to the characteristic boson frequencies $(60 \mathrm{meV} \sim 480$ $\mathrm{cm}^{-1}$ in our case). To elucidate in general the role of interband transitions we illustrate in Fig. 2] a few paradigmatic examples, where we add to the intraband term with $\lambda_{\mathrm{av}}=0.17$ from Fig. 1 (shown here as dashed black line) 




FIG. 2: (color online) Scattering rate (panel a) resulting from the EDM analysis of the optical conductivity $\sigma(\omega)$ from Eq. (4), and its real part $\sigma_{1}(\omega)$ (panel b). The intraband term is computed here with $\lambda_{\mathrm{av}}=0.17$ and the same microscopic parameters as in Fig. 1 The Lorentzian parameters are: (1) $\omega_{L}=1500 \mathrm{~cm}^{-1}, \gamma_{L}=400 \mathrm{~cm}^{-1}, S_{L}=2400 \mathrm{~cm}^{-1} ;(2)$ $\omega_{L}=3000 \mathrm{~cm}^{-1}, \gamma_{L}=400 \mathrm{~cm}^{-1}, S_{L}=2400 \mathrm{~cm}^{-1} ;(3)$ $\omega_{L}=3000 \mathrm{~cm}^{-1}, \gamma_{L}=8000 \mathrm{~cm}^{-1}, S_{L}=2400 \mathrm{~cm}^{-1} ;$ $\omega_{L}=3000 \mathrm{~cm}^{-1}, \gamma_{L}=8000 \mathrm{~cm}^{-1}, S_{L}=24000 \mathrm{~cm}^{-1}$.

a Lorentzian contribution, with a characteristic energy $\omega_{L}$, with weight $S_{L}^{2}$ and width $\gamma$, which mimics interband transitions:

$$
\sigma(\omega)=\sigma_{\text {intra }}(\omega)+\frac{S_{L}^{2}}{4 \pi} \frac{\omega}{\omega \gamma_{L}+i\left(\omega_{L}^{2}-\omega^{2}\right)} .
$$

As one can see in Fig. $2 \mathrm{a}$, the effects on $\tau^{-1}(\omega)$ depend crucially on the weight of the Lorentzian and on its relative width $\gamma_{L} / \omega_{L}$. For $\gamma_{L} / \omega_{L} \ll 1$ (cases 1, 2 in Fig. 2) the Lorentzian peak can be clearly identified in the real part of the conductivity, and it also gives a sharp signature in $\tau^{-1}(\omega)$. However, when $\gamma_{L} / \omega_{L} \simeq 1$ (cases 3,4$)$ the interband contribution adds smoothly to the intraband part, see Fig. 2 $\mathrm{b}$. This leads in turn to an overall smooth increase of the inverse lifetime, that is quantitatively more pronounced when $S_{L}^{2}$ becomes comparable to the spectral weight $\omega_{P}^{2}$ of the intraband part $\left(\omega_{P}=14900\right.$ $\mathrm{cm}^{-1}$ as in Fig. 1). As a consequence, in this case the EDM analysis of the overall conductivity would lead to a misinterpretation of the effects of the interaction, since the dramatic increase of $\tau^{-1}(\omega)$ due to the interband transitions could be interpreted as due to interactions. As we shall argue below, this could be indeed the case in LaFePO.

To establish the effective role of interband transitions in $\mathrm{LaFePO}$ we first estimate their strength and position by computing its complex optical conductivity using Density Functional Theory (DFT) in the generalized gradient approximation (GGA) $\stackrel{25}{=}$ We employed the full potential linearized augmented planewave (LAPW) method



FIG. 3: (color online) (a) Real part of the interband optical conductivity of LaFePO evaluated in DFT calculations. (b)(d) Low-energy band-structure of LaFePO, where the thickness of the symbols represents the character $|m|=0,1,2$ of each band.

as implemented in the Wien2k code $\stackrel{26,27,29}{2}$ The Brillouin zone sampling for the optical calculations was done using a very dense grid of more than $5000 \mathrm{k}$-points in the symmetry irreducible wedge. The complex interband optical conductivity $\sigma(\omega)$ was evaluated using a broadening $\eta=1 \mathrm{meV}$. We considered experimental values for both the lattice constants $(a=3.964 \AA, c=8.512 \AA)$ and the internal coordinate $z_{P}=0.3661$. The interband contribution to $\sigma_{1}(\omega)$ is shown in panel (a) of Fig. 3. Our results are in excellent agreement at all energies with the DFT spectra in literature. $\stackrel{4}{*}$ For $\omega<6000 \mathrm{~cm}^{-1}$, the spectrum shows two main features: a narrow peak at very low energies $\left(\omega \approx 200-600 \mathrm{~cm}^{-1}\right)$, and a broad structure starting at $\omega \approx 2500 \mathrm{~cm}^{-1}$. Note en passant that, according to the above discussion regarding the effects of a Lorentzian interband term illustrated in Fig. 2, the broad high-energy feature in Fig. $3 \mathrm{a}$ is expected to have a considerable effect on the inverse lifetime, while the low-energy peak is expected to produce only modest effects.

The microscopic origin of the two features in the DFT optical conductivity is shown in the bottom panels of Fig. 3. where we decorate the bands of $\mathrm{LaFePO}$ with partial orbital characters. In an energy range $\sim 1 \mathrm{eV}$ around the Fermi level, the bands have mainly Fe $d$ character; $; 2$ optical transitions are possible for $\Delta m= \pm 1$, i.e. between $m=0\left(d_{z^{2}}\right)$ and $|m|=1\left(d_{x z}, d_{y z}\right)$, or between $|m|=1$ and $|m|=2\left(d_{x^{2}-y^{2}}, d_{x y}\right)$ : $\stackrel{29}{\underline{ }}$ The narrow low-energy peak is mostly due to the transitions around the $\Gamma-\mathrm{X}$ and Z-R between the three-dimensional $d_{z^{2}}$ band with $m=0$ and the two-dimensional hole bands with character $d_{x z}, d_{y z}$ $(|m|=1)$. Instead, the higher energy feature mainly involves transitions along $\Gamma$-Z between $|m|=1$ states above the Fermi level and $|m|=2$ states below it. While the position and shape of the first peak is very sensitive 


\begin{tabular}{|c|c|c|c|c|c|c|}
\hline \hline$S_{1}$ & $\omega_{1}$ & $\gamma_{1}$ & $S_{2}$ & $\omega_{2}$ & $\gamma_{2}$ & $\epsilon_{\text {high }}$ \\
\hline 2300 & 750 & 40 & 33500 & 4500 & 20200 & 15 \\
\hline
\end{tabular}

TABLE I: Interband parameters of the model (5) expressed in units of $\mathrm{cm}^{-1}\left(S_{i}, \omega_{i}, \gamma_{i}\right)$ and in dimensionless units $\left(\epsilon_{\mathrm{high}}\right)$.

to computational details and changes for different materials, the second, broad feature, which extends up to $16000 \mathrm{~cm}^{-1}$, is a robust property of the optical spectra of Fe pnictides and chalchogenides $30-34$

It should be remarked however that, although DFT calculations provide an important insight into the existence and nature of low-energy interband transitions we cannot use directly the DFT data to fit the spectrum of Ref. 4. In fact, due to the underestimation of electronic correlations, DFT is known to locate the Fe $d-d$ transitions at larger energy as compared to experiments $\underline{30}$. For instance, in Ref. 33, a direct comparison of experimental and DFT optical spectra of the $\mathrm{Ba} 122$ compound has shown that the experimental position of the Fe $d$ transitions is rescaled by a factor of two with respect to DFT calculations.

Since the exact scaling factor for LaFePO is not known, in order to assess quantitatively the role of interband transitions on the spectra of Ref. 4, we model the infrared interband conductivity with two Lorentzian peaks at low energies,

$$
\sigma(\omega)=\sigma_{\text {intra }}(\omega)+\sum_{i=1,2} \frac{S_{i}^{2}}{4 \pi} \frac{\omega}{\omega \gamma_{i}+i\left(\omega_{i}^{2}-\omega^{2}\right)},
$$

plus a high-energy dielectric constant $\epsilon_{\text {high }}$ to account for processes at higher energies. The intraband part is evaluated for the same microscopic parameters as in Fig. 1 and for $\lambda_{\mathrm{av}}=0.17$, which reproduces the correct experimental behavior of the low-energy Drude-like part of $\sigma_{1}(\omega)$ for $\omega \lesssim 500 \mathrm{~cm}^{-1}$ (see Fig. 1). The Lorentzian parameters are meant instead to model the two groups of transitions shown by DFT calculations. The specific values for the frequency $\left(\omega_{i}\right)$, width $\left(\gamma_{i}\right)$ and weight $\left(S_{i}\right)$ of the two Lorentzian contributions, chosen to fit the experimental data for $\sigma_{1}(\omega)$ in the range $\omega \in[500: 2000]$ $\mathrm{cm}^{-1}$, are reported in Table I.

The result for the total optical conductivity is shown in black line in Fig. 忹, where one can see the very good agreement with the experimental data from Ref. 4 in the whole range $\omega \in[200: 3000] \mathrm{cm}^{-1}$. The effect of the interband transitions on the EDM analysis is elucidated in Fig. 4b where we compare $\tau^{-1}(\omega)$ calculated by using both the total optical response (dashed line) and the interacting intraband part only (solid line). As one can see in Fig. 4 $\mathrm{b}$, the presence of interband transitions remarkably affects the magnitude and the frequency dependence of the effective EDM functions of LaFePO at very low energies $\left(\omega \gtrsim 500 \mathrm{~cm}^{-1}\right)$, in analogy with the case (4) of Fig. 2 discussed above. In particular, the inverse lifetime $\tau^{-1}(\omega)$ acquires a frequency dependence that is
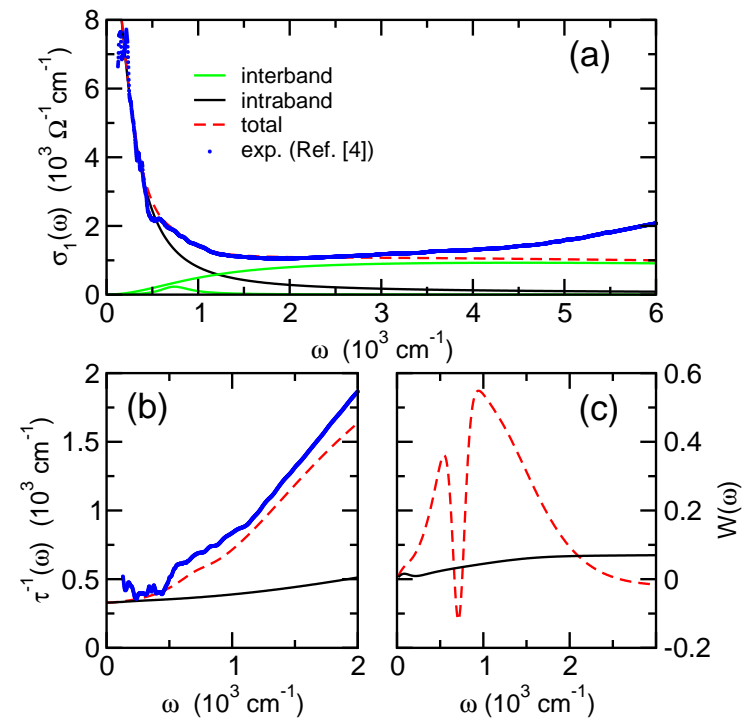

FIG. 4: (color online) (a) Real part of the optical conductivity $\sigma_{1}(\omega)$ computed using Eq. (5) (red dashed line, labeled as "total" in the caption), along with the intraband part (solid black line) and the interband part (solid green line). The symbols represent the experimental data of Ref. 4. (b) $\tau^{-1}(\omega)$ extracted from the extended-Drude-model using the total conductivity (dashed red line) or the intraband part only (solid black line), along with the experimental data (symbols) (c) Approximate electron-boson Eliashberg function $W(\omega)$ extracted from $\tau^{-1}(\omega)$ with the second-derivative method (see text) using either the total conductivity (dashed red line) or the intraband part only (solid black line).

much stronger than the one of the intraband contribution alone, with a linear slope which is $\approx 4$ times larger than the one arising from the actual intraband contribution.

In the spirit of the EDM, the frequency dependence of $\tau^{-1}(\omega)$ is often used to extract the underlying spectrum $\alpha^{2} F(\omega)$ of the retarded interaction supposed to generate it. A crude approximation to obtain the interaction spectrum is the second derivative method, in which $\alpha^{2} F(\omega)$ is approximated by $W(\omega)$, where $W(\omega)=$ $(1 / 2 \pi) d^{2}\left[\omega \tau^{-1}(\omega) / d \omega^{2}\right] \stackrel{35,36}{5}$ This method is usually sufficient to capture the order of magnitude of the spectrum, although not its fine details. The $W(\omega)$ corresponding to the total and to the pure intraband $\tau^{-1}(\omega)$ are shown as dashed red and solid black lines, respectively, in Fig. 卧, showing that the spectrum extracted from the total $\tau^{-1}(\omega)$ is significantly larger than the other one. Indeed, the "effective" coupling $\lambda_{\text {eff }}=2 \int_{0}^{\omega_{c}} d \omega W(\omega) / \omega$, with $\omega_{c}=3000 \mathrm{~cm}^{-1}$, obtained from the intraband term results $\lambda_{\text {eff }}=0.27$, larger but of the same order of magnitude as the microscopic value used here, $\lambda_{\mathrm{av}}=0.17, \underline{37}$ whereas the effective coupling estimated using the total $\tau^{-1}(\omega)$ would give $\lambda_{\text {eff }}=1.31$, significantly larger than $\lambda_{\text {av }}$. This observation can thus explain why a direct EDM analysis of optical data, without a proper subtraction of the interband processes, gives much larger values of the 
coupling ${ }^{5,6}$ than what estimated by other probes, $16,23,24$

In summary, we have shown that the EDM analysis in pnictide systems can be strongly biased by the presence of significant interband contributions in the mid-infrared region of the optical spectrum. In particular, the strong increase of the inverse lifetime $\tau^{-1}(\omega)$, observed in several compounds, $\stackrel{4-8}{-}$ cannot be straightforwardly attributed to strong-coupling effects, as it would be the case for optical spectra dominated by intraband interactions. Even though we focused explicitly on LaFePO, we expect similar effects to hold in other classes of pnictides as well, due to the very similar band structure. In particular, the presence of low-energy interband transitions can provide an alternative explanation for the optical spectra of 122 compounds, where the flat region of the $\sigma_{1}(\omega)$ has been usually fitted with a Drude component having an unrealistically large scattering rate. ${ }^{7,38}$

\section{Acknowledgments}

We thank D. Wu for many useful discussions, and the authors of Ref. 4 for providing us with the experimental data for comparison. L. Boeri. and L. O. would like to thank O. Dolgov, A. Boris and A. Charnukha for useful discussions, and for suggesting us Refs. 2.3. L. O. acknowledges funding from DFG SPP 1458, proj. Bo-3536/1. L.B. and E.C. acknowledge partial funding from the Italian MIUR under the project PRIN 2008XWLWF9.

While completing the present manuscript, we became aware of a work, which discusses experimentally the role of low-energy interband transitions on the optical scattering of $\mathrm{K}$-doped $\mathrm{BaFe}_{2} \mathrm{As}_{2}{ }^{39}$.
1 D.N. Basov and T. Timusk, Rev. Mod. Phys. 77, 721 (2005); D.N. Basov, R. D. Averitt, D. van der Marel, M. Dressel and K. Haule, Rev. Mod. Phys. (2011).

2 S.V. Shulga, Electron-boson effects in the infrared properties of metals, in High-T $T_{c}$ Superconductors and Related Materials, ed. by S.-L. Drechsler and T. Mishonov (Kluwer Academic Publishers, 2001).

3 S.V. Shulga, O.V. Dolgov, and E.G. Maximov, Physica C 178, 266 (1991).

4 M.M. Qazilbash, J.J. Hamlin, R.E. Baumbach, L. Zhang, D.J. Singh, M.B. Maple, and D.N. Basov, Nature Physics 5, 647 (2009).

5 J. Yang, D. Huvonen, U. Nagel, T. Room, N. Ni, P.C. Canfield, S.L. Budko, J.P. Carbotte, and T. Timusk, Phys. Rev. Lett. 102, 187003 (2009).

${ }^{6}$ D. Wu, N. Barišić, M. Dressel, G.H. Cao, Z-A. Xu, E. Schachinger, and J.P. Carbotte, Phys. Rev. B 82, 144519 (2010).

7 J.J. Tu, J. Li, W. Liu, A. Punnoose, Y. Gong, Y.H. Ren, L.J. Li, G.H. Cao, Z.A. Xu, and C.C. Homes, Phys. Rev. B 82, 174509 (2010).

8 N. Barišić, D. Wu, M. Dressel, L.J. Li, G.H. Cao, and Z.A. Xu, Phys. Rev. B 82, 054518 (2010).

9 I.I. Mazin, D.J. Singh, M.D. Johannes, and M.H. Du, Phys. Rev. Lett. 101, 057003 (2008); K. Kuroki, S. Onari, R. Arita, H. Usui, Y. Tanaka, H. Kontani, and H. Aoki, Phys. Rev. Lett. 101, 087004 (2008).

10 L. Benfatto, E. Cappelluti, and C. Castellani Phys. Rev. B 80, 214522 (2009)

11 F. Hardy, P. Burger, T. Wolf, R. A. Fisher, P. Schweiss, P. Adelmann, R. Heid, R. Fromknecht, R. Eder, D. Ernst, H. v. Löhneyse,n and C. Meingast, Europhys. Lett. 9147008 (2010).

12 J. G. Analytis, J.-H. Chu, R. D. McDonald, S. C. Riggs, and I. R. Fisher, Phys. Rev. Lett. 105, 207004 (2010).

13 E. van Heumen, Y. Huang, S. de Jong, A.B. Kuzmenko, M.S. Golden, and D. van der Marel, Europhys. Lett. 90, 37005 (2010).

14 S.-L. Drechsler, M. Grobosch, K. Koepernik, G. Behr, A. Köhler, J. Werner, A. Kondrat, N. Leps, C. Hess, R. Klingeler, R. Schuster, B. Büchner, and M. Knupfer,
Phys. Rev. Lett. 101, 257004 (2008); S.-L.Drechsleret al. arXiv:0904.0827.

15 A.V. Boris, N.N. Kovaleva, S.S.A. Seo, J.S. Kim, P. Popovich, Y. Matiks, R.K. Kremer, and B. Keimer, Phys. Rev. Lett. 102, 027001 (2009).

16 L. Ortenzi, E. Cappelluti, L. Benfatto, and L. Pietronero, Phys. Rev. Lett. 103, 046404 (2009).

17 L. Benfatto and E. Cappelluti, Phys. Rev. B 83, 104516 (2011).

18 D.S. Inosov, J.T. Park, P. Bourges, D.L. Sun, Y. Sidis, A. Schneidewind, K. Hradil, D. Haug, C.T. Lin, B. Keimer, and V. Hinkov, Nat. Phys. 6178 (2010).

19 L. Boeri, O.V. Dolgov, and A.A. Golubov Phys. Rev. Lett. 101, 026403 (2008).

20 A.J. Millis, Phys. Rev. B 45, 13047 (1992).

21 Y. Kamihara, H. Hiramatsu, M. Hirano, R. Kawamura, H. Yanagi, T. Kamiya, and H. Hosono, J. Am. Chem. Soc. 128, 10012 (2006).

22 A.I. Coldea, J.D. Fletcher, A. Carrington, J.G. Analytis, A.F. Bangura, J.-H. Chu, A.S. Erickson, I.R. Fisher, N.E. Hussey, and R.D. McDonald, Phys. Rev. Lett. 101, 216402 (2008).

23 T.M. McQueen, M. Regulacio, A.J. Williams, Q. Huang, J.W. Lynn, Y.S. Hor, D.V. West, M.A. Green, and R. J. Cava, Phys. Rev. B 78, 024521 (2008).

${ }^{24}$ Y. Kohama, Y. Kamihara, H. Kawaji, T. Atake, M. Hirano, and H. Hosono, J. Phys. Soc. Jpn. 77, 094715 (2008).

25 J. P. Perdew, K. Burke, and M. Ernzerhof, Phys. Rev. Lett. 77, 3865 (1996).

26 O. K. Andersen, Phys. Rev. B 12, 3060 (1975).

27 http://www.wien2k.at.

28 S. Lebègue, Phys. Rev. B 75, 035110 (2007).

29 C. Ambrosch-Draxl and J. Sofo, Comp. Phys. Comm. 175, 1 (2006).

${ }^{30}$ K. Haule, J.H. Shim and G. Kotliar, Phys. Rev. Lett. 100, $226402(2008)$.

31 J. Ferber, Y.-Z. Zhang, H.O. Jeschke, and R. Valentí, Phys. Rev. B 82, 165102 (2010)

32 A. Sanna, F. Bernardini, G. Profeta, S. Sharma, J.K. Dewhurst, A. Lucarelli, L. Degiorgi, E.K.U. Gross, and S. Massidda, Phys. Rev. B 83, 054502 (2011). 
33 A. Charnukha, P. Popovich, Y. Matiks, D.L. Sun, C.T. Lin, A.N. Yaresko, B. Keimer, and A. V. Boris, Nat. Comm. 2, 219 (2011).

34 Z.P. Yin, K. Haule, and G. Kotliar, Nat. Phys. 7, 294 (2011).

35 M.R. Norman and A.V. Chubukov, Phys. Rev. B 73, 140501 (2006).

36 E. Schachinger, D. Neuber, and J.P. Carbotte, Phys. Rev. B 73, 184507 (2006).

${ }^{37}$ For the pure intraband case, moderate deviation from $\lambda_{\text {eff }}$ and $\lambda_{\text {av }}$ are expected to arise due to the finite temperature effects and due to the failure, in multiband systems, of the Allen approximation used to define the second derivative method.

38 D. Wu, N. Barišić, P. Kallina, A. Faridian, B. Gorshunov, N. Drichko, L.J. Li, X. Lin, G.H. Cao, Z.A. Xu, N.L. Wang, and M. Dressel, Phys. Rev. B 81, 100512(R) (2010).

39 A. Charnukha, O.V. Dolgov, A.A. Golubov, Y. Matiks, D.L. Sun, C.T. Lin, B. Keimer, and A.V. Boris, arXiv:1103.0938 